\title{
Corrigendum to: Influence of road traffic noise peaks on reading task performance and disturbance in a laboratory context
}

\author{
Catherine Lavandier ${ }^{1, *}$ (1), Mehdi Regragui ${ }^{1,2}$, Romain Dedieu ${ }^{1}$ (D), Carine Royer ${ }^{3}$ (1), and Arnaud Can $^{2}$ (1) \\ ${ }^{1}$ Laboratoire ETIS, CY Cergy Paris University, ENSEA, CNRS, UMR8051, 95000 Cergy, France \\ ${ }^{2}$ UMRAE, Univ Gustave Eiffel, IFSTTAR, CEREMA, 44340 Bouguenais, France \\ ${ }^{3}$ Laboratoire Paragraphe EA349, CY Cergy Paris Université, 95000 Cergy, France
}

The online version of the original article was published as: Acta Acustica, 6, 3, https://doi.org/10.1051/aacus/2021057

The authors wish to correct Figure 7, an error appeared in the above manuscript: Figures 7 and 8 were the same. The real Figure 7 is presented below.

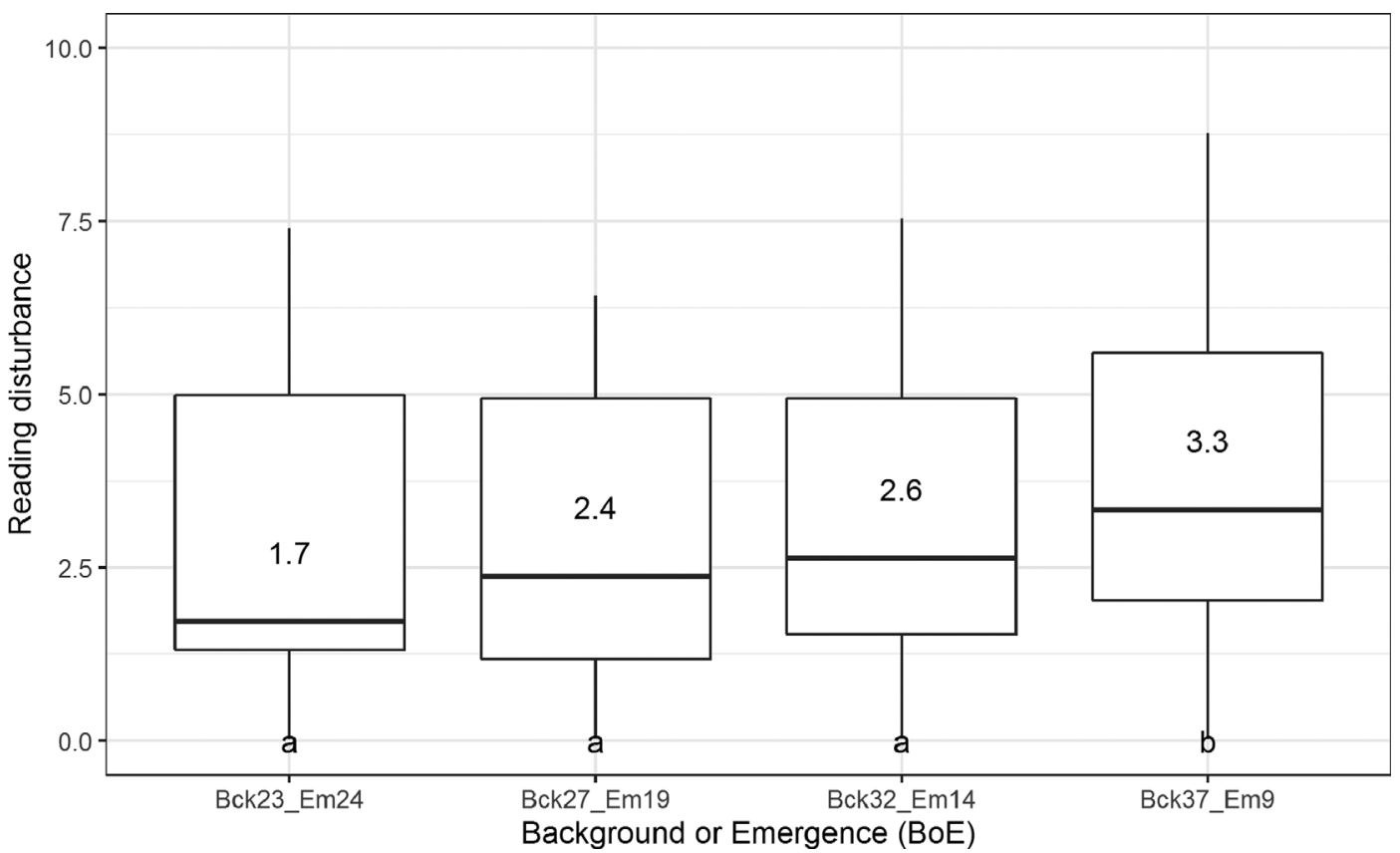

Figure 7. Reading disturbance according to the (BoE) factor only for the group of noise sensitive participants. Labels correspond to median values for each distribution. The two groups a and b, representing significant different distributions, are extracted from the Nemenyi post-hoc analysis.

Cite this article as: Lavandier C. Regragui M. Dedieu R. Royer C. \& Can A. 2022. Corrigendum to: Influence of road traffic noise peaks on reading task performance and disturbance in a laboratory context. Acta Acustica, 6, 7.

*Corresponding author: catherine.lavandier@cyu.fr 